\title{
Clinicopathological Correlation of Adenoid Cystic Carcinoma: A Notorious Masquerader and Clinical Paradox
}

\begin{abstract}
Background: Adenoid cystic carcinoma (ACC) is an uncommon tumor with nonspecific clinicoradiological features thereby masquerading other nonneoplastic and neoplastic entities. Materials and Methods: Cases of ACC were retrospectively reviewed over a period of 4 years. The clinical details of these patients including fine-needle aspiration cytology (FNAC) and imaging findings were retrieved. Diagnosis was confirmed on histomorphology and supplemented with immunohistochemistry (IHC). Results: Thirty cases of ACC were included in the study. Mean patient age was 55.5 years with a slight female preponderance. Among the 30 ACCs, 10 (33.4\%) were located in submandibular gland, $7(23.4 \%)$ in parotid gland, $6(20 \%)$ in sublingual gland, $2(6.7 \%)$ in lung and one each $(3.33 \%)$ in nasal cavity, breast, cervix, lip, and skin of face. Preoperative imaging was suggestive of malignancy in 29 cases while a single case of parotid gland ACC was misdiagnosed as benign salivary gland neoplasm. FNAC was performed in 29 cases with a diagnostic accuracy of $82.7 \%$. Histopathological examination showed characteristic features of ACC in all cases with perineural invasion seen in 7 cases. On IHC, positivity for cytokeratin was seen in all cases, cluster of differentiation 117 in 24 cases, thyroid transcription factor-1 in two cases and human epidermal growth factor receptor/neu in two cases. All cases were negative for estrogen receptor and progesterone receptor IHC. Mean Ki-67 score was 47.8\%. Conclusion: ACCs are notorious tumors showing slow growth kinetics with propensity for perineural invasion, late recurrences, and distant metastasis. It should be kept in mind as a differential diagnosis at unusual sites other than salivary glands.
\end{abstract}

Keywords: Adenoid cystic carcinoma, fine-needle aspiration cytology, perineural, salivary glands

\section{Introduction}

Adenoid cystic carcinoma (ACC) is a relatively uncommon tumor, with a reported incidence of 4.5 cases per million individuals. ${ }^{[1]}$ It most commonly involves the salivary glands. ${ }^{[2]}$ Involvement of lacrimal gland, breasts, uterine cervix, esophagus, lungs, and prostate is exceedingly rare and infrequently reported. ${ }^{[3]}$

Owing to nonspecific clinicoradiological features, these tumors masquerade other nonneoplastic and neoplastic entities. On literature search scattered case reports and case series of site specific ACCs were found without any large study on the incidence, distribution and clinicopathological features of ACC at different sites. Keeping in view these considerations, we retrospectively analyzed cases of ACCs over a period of 4 years in a tertiary care setting. Our study highlights the clinicopathological features

This is an open access journal, and articles are distributed under the terms of the Creative Commons Attribution-Non Commercial-ShareAlike 4.0 License, which allows others to remix, tweak, and build upon the work non-commercially, as long as appropriate credit is given and the new creations are licensed under the identical terms.

For reprints contact: reprints@medknow.com of ACC with special emphasis on the utility of preoperative diagnostic modalities such as imaging and fine-needle aspiration cytology (FNAC).

\section{Materials and Methods}

The archives of Department of Histopathology were retrospectively reviewed from January 2012 to January 2016. Of the 57,890 cases received, cases of ACC were included in the study. The medical records and clinical details of these patients including preoperative FNAC and/or radiological findings on computed tomography (CT)/magnetic resonance imaging (MRI)/mammography were retrieved. FNAC was performed wherever possible. Repeat aspiration was performed if the first FNA attempt yielded inadequate material or was inconclusive for diagnosis. Smears were then air dried and stained with May-Grünwald Giemsa stain. All the patients underwent

How to cite this article: Sharma $P$, Jha $V$, Mandal AK. Clinicopathological correlation of adenoid cystic carcinoma: A notorious masquerader and clinical paradox. Indian J Med Paediatr Oncol 2018;39:276-81.

\section{Preeti Sharma, Vidya Jha, Ashish Kumar Mandal}

Department of Pathology, Vardhman Mahavir Medical College and Safdarjung Hospital, New Delhi, India
Address for correspondence: Dr. Vidya Jha, Department of Pathology, Vardhman Mahavir

Medical College and Safdarjung Hospital, New Delhi - 110 029, India. E-mail: preetisharma261189@ gmail.com

Access this article online Website: www.ijmpo.org DOI: 10.4103/ijmpo.ijmpo_141_16 Quick Response Code:

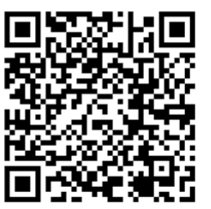


curative surgery. The excised specimen was fixed in $10 \%$ neutral buffered formalin and sent for histopathological examination. Diagnosis was confirmed on hematoxylin and eosin ( $\mathrm{H}$ and $\mathrm{E})$ stained, formalin-fixed paraffin embedded sections. These tumors were graded as per recommendations by Szanto et al. ${ }^{[4]}$ Tumors containing only tubular or cribriform growth pattern were categorized as Grade I tumors, tumors containing cribriform or tubular growth with $<30 \%$ solid component as grade II tumors and tumors containing $>30 \%$ solid component as Grade III tumors. A detailed immunohistochemical (IHC) panel consisting of cytokeratin (CK) (Biocare Medical, Clone: AE1/AE3), cluster of differentiation 117 (CD117) 117 (Biocare Medical, Clone: EP10), thyroid transcription factor-1 (TTF-1) (Biocare Medical, Clone: 8G7G3/1), estrogen receptor (ER) (Thermo Scientific, Clone: SP1), progesterone receptor (PR) (Thermo Scientific, Clone: SP2), human epidermal growth factor receptor (HER2/neu) (BioGenex, Clone EP1045Y), and Ki-67 (Thermo Scientific, Clone: SP6) was applied to all cases. In brief, sections measuring 3-4 $\mu \mathrm{m}$ thick were cut, deparaffinized with xylene and brought to water through graded levels of alcohol. Endogenous peroxidase activity was blocked by treating the slides with hydrogen peroxide for $30 \mathrm{~min}$ at room temperature. Antigen retrieval was done by immersing the slides in citrate buffer using the pressure cooker method. Then, the slides were incubated overnight with the primary antibody (rabbit polyclonal) at $4^{\circ} \mathrm{C}$ in a humidified chamber. The following day secondary antibody was added. The sections were then incubated with di amino benzidine for visualization of the peroxidase reaction. After being washed in water, the sections were counter stained with hematoxylin, dehydrated in alcohol, cleared in xylene, and mounted. IHC was interpreted in a binary fashion as positive or negative. Ki-67 index was calculated by counting the total number of Ki-67 positive cells in 100 consecutive tumor cells.

The FNAC smears, $\mathrm{H}$ and $\mathrm{E}$ stained sections, and IHC slides of all the cases were reviewed by two pathologists and correlated with the clinical findings including radiology.

\section{Results}

Out of 57,890 cases received in the department over a period of 4 years, we found only 30 cases of ACC [Table 1]. The mean patient age at the time of surgery was 55.5 years (range 19-64 years). There were 13 male and 17 female patients with a male:female ratio of $1: 1.3$. Among these 30 tumors, $10(33.4 \%)$ were located in the submandibular gland, $7(23.4 \%)$ in the parotid gland, $6(20 \%)$ in the sublingual gland, $2(6.7 \%)$ in the lung and one each $(3.33 \%)$ in nasal cavity, breast, cervix, lip, and skin of face.

Clinical presentation of these patients was variable and site dependent. All cases of ACC in head and neck region (26 cases) presented with complaints of a slow

\begin{tabular}{lc}
\hline \multicolumn{2}{c}{ Table 1: Site distribution of $\begin{array}{c}\mathbf{3 0} \text { cases of adenoid cystic } \\
\text { carcinoma }\end{array}$} \\
\hline Site & Number of cases (\%) \\
\hline Submandibular gland & $10(33.4)$ \\
Parotid gland & $7(23.4)$ \\
Sublingual gland & $6(20)$ \\
Lung & $2(6.7)$ \\
Breast & $1(3.33)$ \\
Cervix & $1(3.33)$ \\
Nasal cavity & $1(3.33)$ \\
Lip & $1(3.33)$ \\
Cutaneous (face) & $1(3.33)$ \\
Total & 30 \\
\hline
\end{tabular}

growing painless mass associated with facial pain in two cases and a single case with recurrent epistaxis. Apart from head and neck, a 35-year-old female patient presented with bleeding on and off for 5 months while an elderly female presented with a firm immobile breast lump for 8 months. Two female patients presented with prolonged cough and shortness of breath lasting for 1 year.

Preoperative imaging was performed in all 30 cases [Figure 1a-f]. Contrast-enhanced CT (CECT) findings were suggestive of a malignant tumor in 28 cases and depicted the extent of the primary lesion accurately. However, definite categorization of the malignant tumors could not be established in any of these cases. A single case of parotid gland ACC was misdiagnosed as a benign salivary gland neoplasm on CECT [Figure 1a]. Mammography findings in an elderly female with a breast lump were suggestive of malignancy (BIRADS IV) [Figure 1f]. Metastatic deposits in the lung were reported in one patient with submandibular ACC and one case of sublingual ACC. However, lymph node metastasis was seen in only a single patient with sublingual ACC.

FNAC was performed in all cases except for the mass in uterine cervix due to inaccessibility of lesion. Further, CT-guided FNAC was performed for the lung lesions. FNA smears were moderately cellular with cells arranged in variably sized cohesive clusters and occasional cup shaped fragments [Figure 2a]. The individual tumor cells were small with scant basophilic cytoplasm and central round hyperchromatic nucleus. Numerous variable sized, spherical, and pink hyaline globules were seen both within and outside the cell clusters [Figure 2b]. Accurate diagnosis of ACC was made in $82.7 \%$ cases (24/29 cases) on FNAC. In the remaining, two cases of parotid gland swelling and one case of swelling in the submandibular region yielded paucicellular smears after repeated attempt and were inconclusive for a definite opinion. However, aspirate smears from one of the swellings on the floor of mouth and peripheral lung nodule yielded hemorrhagic smears and was inadequate for diagnosis. Interestingly, a 55-year-old female presented with a firm, slow growing, ill-defined 

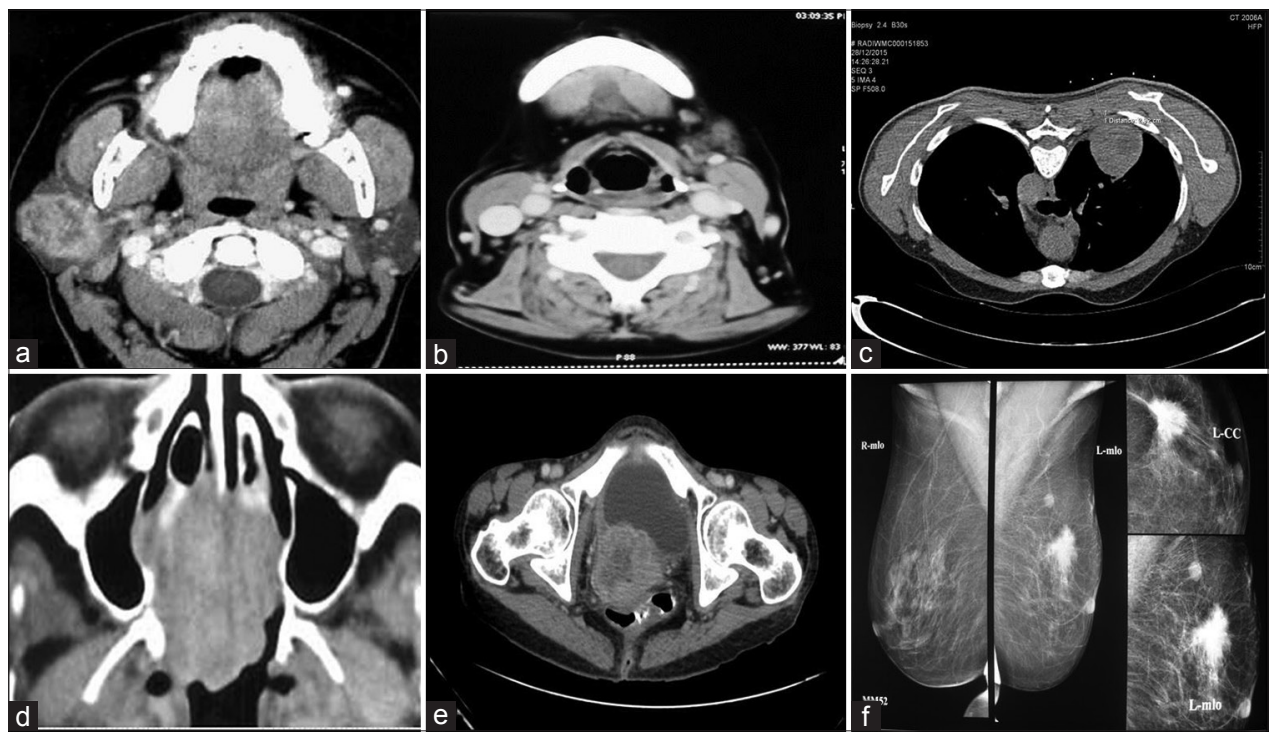

Figure 1: Contrast enhanced computed tomography image shows (a) Heterogeneously enhancing mass lesion involving superficial and deep lobes of right parotid gland, (b) Small lobulated mass lesion just anterior to the left submandibular gland with areas of necrosis, (c) Heterogeneously enhancing mass in posterior segment of right upper lobe, (d) Homogeneous mass involving the nasal septum along with bony erosion of medial wall of right orbit, (e) Heterogeneously enhancing cervical mass seen obstructing the endocervical canal, (f) Breast mammography image shows a spiculated mass in upper outer quadrant of left breast suggestive of BIRADS IV

swelling on the floor of month for 1 year. Simultaneously, she also complained of a breast lump in the same duration. FNA smears from the sublingual swelling revealed features of ACC while aspirate smears from breast lump showed infiltrating ductal carcinoma, suggestive of synchronous breast and minor salivary gland tumor.

Surgical resection specimens in all patients showed a mean tumor size of $3.2 \mathrm{~cm} . \mathrm{H}$ and $\mathrm{E}$ stained sections showed cribriform and tubular arrangement of tumor cells forming pseudocysts filled with basement membrane-like basophilic material. The individual tumor cells were round to cuboidal with scant eosinophilic cytoplasm and central hyperchromatic nucleus [Figure $2 \mathrm{c}$ and d]. As per the grading system, 25 cases belonged to Grade I, 4 cases to Grade II and one case to grade III. Perineural invasion was seen in seven cases (33.33\%). On IHC, all the cases showed cytoplasmic positivity for CK [Figure 3a]. Membranous and cytoplasmic positivity for CD117 was seen in 24 cases $(80 \%)$ [Figure 3b]. TTF-1 was positive in two cases of pulmonary ACC only [Figure 3c]. Focal HER2/neu immunopositivity was seen in two cases of parotid gland ACC while ER and PR expression was not observed in any of the 30 cases. ACC of breast was thereby categorized as a triple-negative breast cancer. Mean Ki-67 score of the 30 cases studied was $47.8 \%$ [Figure $3 \mathrm{~d}$ ].

\section{Discussion}

Although ACC was first described in 1853, the term was denominated by Spies later in $1930 .^{[5]}$ The usual peak incidence of ACC is between 50 and 60 years of age with a slight female preponderance, ${ }^{[6]}$ and our results were in concordance with published literature. ACC comprises $10 \%$ of all salivary gland tumors and most commonly affects the submandibular gland followed by parotid gland. ${ }^{[7]}$ In the present series, similar results were obtained along with localization at unusual sites such as lung, palate, breast, cervix, and face.

Among the rare cases, ACC of the breast previously known as cylindroma accounts for $<0.1 \%$ of all primary breast cancers ${ }^{[8]}$ The major histopathological differential diagnosis for ACC includes cribriform carcinoma of breast. However, the latter shows epithelial cells only without basement membrane-like material. While ACC is categorized as basal - like subtype of breast carcinoma, cribriform carcinoma usually expresses ER and PR positivity on IHC staining. ${ }^{[9]}$ Due to scarcity of reported cases and variations in the patterns of this tumor, no definite guidelines for treatment have been established till date. Borrowing from literature the outcome of these tumors is favorable vis-à-vis other forms of breast cancer and ACCs of the salivary glands..$^{[10]}$

The cell of origin for ACC of cervix is proposed to be the "reserve cells" in the endocervix where it comprises $<1 \%$ of all cervical carcinoma ${ }^{[11,12]}$ Although the exact pathogenesis remains elusive, the role of human papillomavirus in ACC has been suggested in the past. On the contrary, pulmonary ACC of lung is an unusual neoplasm arising from the submucosal glands of the tracheobronchial tree and accounts for $0.04-0.2 \%$ of all primary pulmonary tumors. ${ }^{[13]}$ The large airways are most commonly involved in over $80 \%$ of cases. ${ }^{[14]}$ Our study included two cases of pulmonary ACC one of which was located peripherally and yielded hemorrhagic smears of FNAC. The second case of pulmonary ACC was located along the main bronchus, and 


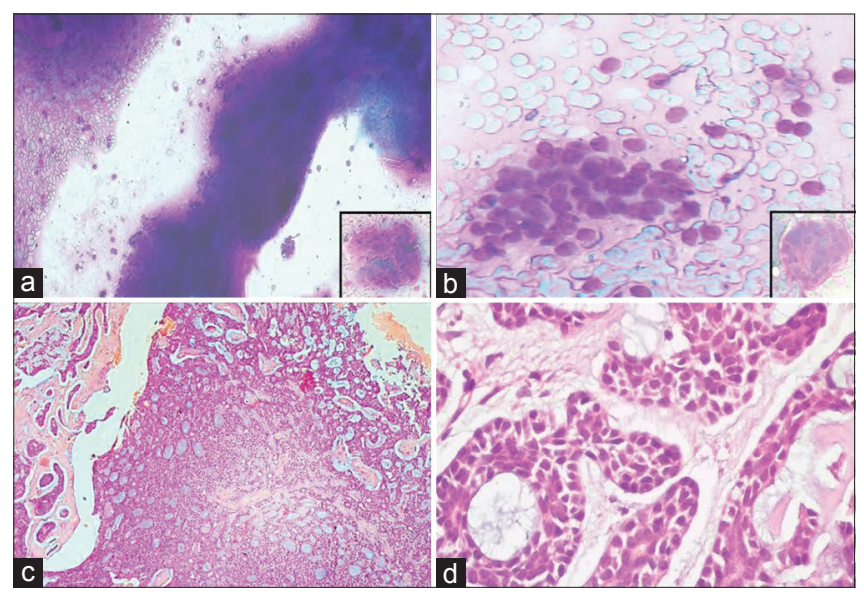

Figure 2: Photomicrograph of May Grünwald Giemsa stained fine needle aspiration smears show; (a) Moderate cellularity with small basaloid cells arranged in cohesive clusters and occasional cup shaped fragments (inset) (×200). (b) Tumor cells are embedded within spherical, pink hyaline globules (inset) $(\times 200)$. Photomicrograph of $\mathrm{H}$ and $\mathrm{E}$ stained sections show; (c) Cribriform arrangement of tumor cells forming pseudocysts filled with basement membrane like basophilic material $(\times 100)$. (d) Tubular arrangement of cells which are round to cuboidal with scant eosinophilic cytoplasm and central hyperchromatic nucleus $(\times 400)$

CT-guided FNA smears were diagnostic of ACC. Although aspirate smears usually yield characteristic features of ACC comprising basaloid cells arranged in clusters and embedded within hyaline globules, the cytomorphological differential diagnosis in doubtful cases includes carcinoid tumor, small cell carcinoma, and well-differentiated adenocarcinoma. The absence of hyaline globules and glandular architecture excludes the diagnosis of carcinoid tumors while clusters of uniform cells with glandular arrangement are characteristic of well-differentiated adenocarcinoma. Small cell carcinoma on the contrary is characterized by nuclear molding and nuclear streaking. ${ }^{[15]}$

Nasal cavity and skin are other unusual sites for ACC with $<70$ cases of the latter described in English literature. ${ }^{[16,17]}$ Histogenesis of primary cutaneous ACC is still not clear and no universally agreed predisposing factor is known. Clinical assessment of all of the reported cutaneous ACCs led to a misdiagnosis of a skin appendageal tumor.

ACC is a clinical masquerader and includes a varied spectrum of lesions in the differential diagnosis clinically. Pleomorphic adenoma, mucoepidermoid carcinoma, and polymorphous low-grade adenocarcinoma are usually considered in the setting of salivary gland involvement. At the unusual locations mentioned earlier, clinical diagnosis of this notorious tumor remains a diagnostic dilemma.

A rather interesting and unusual case of dual primary malignancies was encountered in our series. A 55-year-old female was simultaneously diagnosed with ACC of floor of the mouth and infiltrating ductal carcinoma of the breast. The presence of multiple primary malignancies in a patient can either be detected at the same time (synchronous),

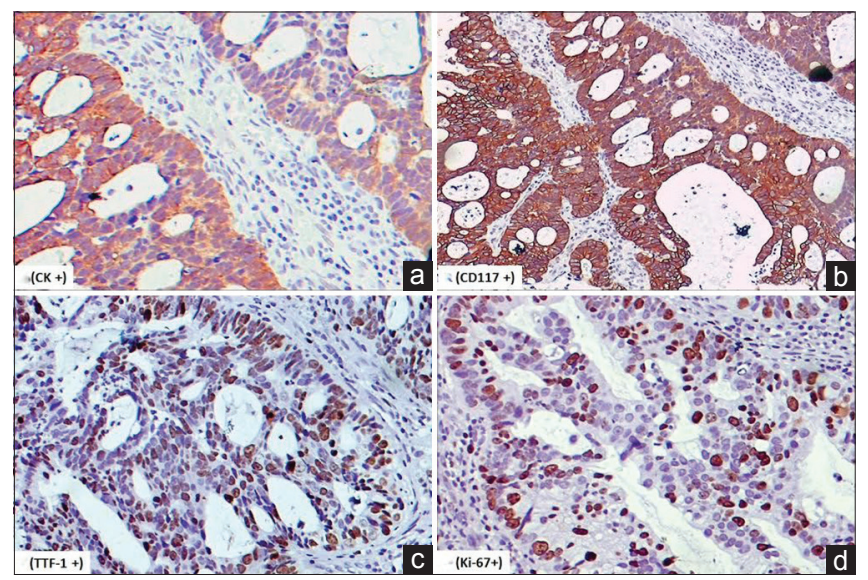

Figure 3: On immunohistochemistry tumor cells were positive for; (a) cytokeratin (immunostain, $\times 200$ ), (b) cluster of differentiation 117 (immunostain, $\times 200$ ), (c) thyroid transcription factor-1 (immunostain, $\times 200$ ), (d) Ki-67 (immunostain, $\times 200$ )

or one cancer may follow the other after a certain period (metachronous). If the initial primary is the breast, the percentage of patients expected to develop multiple primaries is $10 \% \cdot{ }^{[18]}$ Dual primary malignancies have been identified with increasing frequency, possibly due to the increase in life expectancy of cancer survivors and more robust cancer screening protocols. The causal factors for this occurrence include persistent carcinogen exposure, ionizing radiation, and the increasing use of hormonal therapies, genetic manipulation, and immunomodulators. ${ }^{[19]}$ However, the exact causal relationship of the dual malignancies in our patient could not be ascertained. This particular case is the first case of dual primary malignancy, i.e., ACC of floor of mouth and infiltrating ductal carcinoma of breast in a single patient, thereby highlighting that presence of a lesion anatomically away from the primary malignancy should be labeled as a metastatic focus only after detailed evaluation including histopathological confirmation.

Preoperative diagnosis of ACC is a challenging task for the radiologists due to nonspecific signs. CT scan helps in localization of the lesions and delineates the extent of malignancy. In our study, CT scan identified malignant masses in all cases but was unable to categorize the lesion in any. On the other hand, FNAC is a simple, minimally invasive, and cost-effective first line outpatient department investigation which can render a preoperative diagnosis of ACC. However, intraoral lesions pose a diagnostic difficulty owing to the inaccessibility and technical difficulty in performing mucosal FNAC and often yield hemorrhagic/inadequate aspirates as seen in two of our cases. Thus, the diagnosis of ACC is solely based on morphology. However, morphology alone cannot differentiate primary from metastatic ACCs and a complete clinicoradiological work up is mandatory to rule out any focus of primary tumor elsewhere.

The expression of IHC markers has been evaluated as potential prognostic indicators in ACC. Proliferation 
marker Ki-67 comes across as a promising immunostain showing an increased expression with increasing amounts of solid (grade III) component and a definite correlation with worse prognosis. ${ }^{[20,21]}$ CD117 (c-KIT) immunoreactivity has also been evaluated in a variety of tumors due to the presence of targeted inhibitor therapy against it. KIT positivity has been commonly observed in gastrointestinal stromal tumors and seminoma, rare cases of synovial sarcoma and Ewing sarcoma, as well as melanocytic tumors like nevi and malignant melanomas. However, its expression in carcinomas is rather rare, with the exception of small cell lung carcinoma and ACC. ${ }^{[22]}$ In our series, positivity for CD117 was seen in $80 \%$ of cases. Since our study was a retrospective analysis, inability to analyse c-KIT mutation and response to inhibitor therapy was a drawback. Similar to CD117, targeted therapy against HER2/neu makes it a valuable marker. However, low prevalence of HER2/neu overexpression in ACC as also seen in our study limits the clinical utility of Herceptin therapy. ${ }^{[23]}$

Another immunostain studied was TTF-1. Expression of this marker is observed in both normal and neoplastic lung and thyroid tissues. Similar to publisher data, only two cases of pulmonary ACC showed positivity for this marker in contrast to other sites. Therefore, TTF-1 is considered as a reliable marker of pulmonary and in the setting of metastasis. Recently, Jungsuk et al. concluded that TTF-1 expression should be interpreted in the context of the clinical setting, radiologic findings as it can be expressed in ACCs metastatic to the lung despite being negative in the primary tumor site. ${ }^{[24]}$

ACC exhibits a paradoxical clinical behavior. Initially, the tumor presents as a slow-growing indolent mass, but the subsequent course is progressive and usually associated with multiple recurrences. Hematogenous spread to distant organs such as lung and bone is well documented with regional nodal involvement being rather rare. ${ }^{[25]}$ Of the 30 cases studied, we found only one patient with lung and regional nodal metastasis each. Fewer cases with distant metastatic foci were observed which may be attributed to a shorter follow-up in.

While tumor stage using the American Joint Committee on Cancer is considered as the most reliable prognostic indicator, ${ }^{[26]}$ some authors have emphasized the importance of histologic subtyping with tubular subtype carrying the best prognosis in contrast to solid subtype. Although the prognostic significance of this grading system has been questioned time and again, ${ }^{[27]}$ the presence of a solid component has been a consistent predictor of poor prognosis in several series. ${ }^{[28]}$ Moreover, there is a strong correlation between site of origin and prognosis. ACC involving the major salivary glands has a more favorable outcome in comparison to minor salivary glands. Localization of ACC in the nasal cavity and paranasal sinuses carries the worse prognosis than in any other area of the head and neck region. ${ }^{[29]}$

Furthermore, the paradoxical behavior of ACC is highlighted by an optimistically high 5-year survival rate in contrast to a dismally low 10-20 year survival rate. ${ }^{[30]}$

Till date, surgery and radiotherapy still remain the main course of treatment. Nonetheless, after resection of the primary tumor, this notorious tumor is associated with local and distant recurrences. This high-recurrence rate likely reflects the known tendency for perineural invasion. Careful documentation of perineural invasion during staging is especially important as it stands as an independent prognostic factor. ${ }^{[18]}$ Various authors have reported duration of recurrence varying from 18 months to 67 months thereby making long-term follow in these patients mandatory. Periodic radiological assessment especially MRI may identify changes indicative of recurrent disease many months before clinical evidence. ${ }^{[10]}$

To conclude, ACC is a malignant tumor with a deceptively benign histologic appearance characterized by indolent, locally invasive growth with high propensity for local recurrence and distant metastasis. Chest examination should invariably be done, as these tumors may have propensity for pulmonary metastasis. Trials of targeted therapy to date have not yet identified an agent with sufficient activity to be deemed standard in the treatment of advanced ACC. The immediate future of research in this field of the molecular pathogenesis of ACC will not only be of diagnostic importance but will also derive targeted therapies for this neoplasm.

\section{Financial support and sponsorship}

Nil.

\section{Conflicts of interest}

There are no conflicts of interest.

\section{References}

1. Bonaparte JP, Hart R, Trites J, Taylor MS. Incidence of adenoid cystic carcinoma in nova scotia: 30-year population-based epidemiologic study. J Otolaryngol Head Neck Surg 2008;37:642-8.

2. Kokemueller H, Eckardt A, Brachvogel P, Hausamen JE. Adenoid cystic carcinoma of the head and neck - A 20 years experience. Int J Oral Maxillofac Surg 2004;33:25-31.

3. Dodd RL, Slevin NJ. Salivary gland adenoid cystic carcinoma: A review of chemotherapy and molecular therapies. Oral Oncol 2006;42:759-69.

4. Szanto PA, Luna MA, Tortoledo ME, White RA. Histologic grading of adenoid cystic carcinoma of the salivary glands. Cancer 1984;54:1062-9.

5. Spies JW. Adenoid cystic carcinoma. Arch Surg 1930;21:365-404.

6. Jaso J, Malhotra R. Adenoid cystic carcinoma. Arch Pathol Lab Med 2011;135:511-5.

7. Chummun S, McLean NR, Kelly CG, Dawes PJ, Meikle D, Fellows S, et al. Adenoid cystic carcinoma of the head and neck. Br J Plast Surg 2001;54:476-80. 
8. Muslimani AA, Ahluwalia MS, Clark CT, Daw HA. Primary adenoid cystic carcinoma of the breast: Case report and review of the literature. Int Semin Surg Oncol 2006;3:17.

9. Chin S, Kim Z. Adenoid cystic carcinoma of the breast: Report of two cases with immunohistochemical profile of C-kit and MYB overexpression. Indian J Pathol Microbiol 2014;57:611-3.

10. Li N, Xu L, Zhao H, El-Naggar AK, Sturgis EM. A comparison of the demographics, clinical features, and survival of patients with adenoid cystic carcinoma of major and minor salivary glands versus less common sites within the Surveillance, Epidemiology, and End Results registry. Cancer 2012;118:3945-53.

11. Maheshwari GK, Dave KS, Wadhwa MK, Gopal U, Shah R. Adenoid cystic carcinoma of the uterine cervix with pulmonary metastasis 11 years after radiotherapy: Case report. Turk J Cancer 2000;30:181-5.

12. Grayson W, Taylor LF, Cooper K. Adenoid cystic and adenoid basal carcinoma of the uterine cervix: Comparative morphologic, mucin, and immunohistochemical profile of two rare neoplasms of putative 'reserve cell' origin. Am J Surg Pathol 1999;23:448-58.

13. Travis WD, Travis LB, Devesa SS. Lung cancer. Cancer 1995; 75:191-202.

14. Kumari S, Saugat R, Kapoor A, Paramanandhan M, Soni G, Sirohi P. Adenoid cysticcarcinoma of lung: A very unusual presentation. Int J Sci Rep 2015;1:143-5.

15. Bhalara RV, Gamit MJ, Popat M, Gandhi SH, Dhruva GA. Cytomorphology of primary adenoid cystic carcinoma of lung: An exceedingly rare case. APALM 2015;2:223-6.

16. Belaldavar BP, Batra R. Adenoid cystic carcinoma of the nasal septum: A rare case report. J Sci Soc 2013;40:39-40.

17. Tonev ID, Pirgova YS, Conev NV. Primary adenoid cystic carcinoma of the skin with multiple local recurrences. Case Rep Oncol 2015;8:251-5.

18. Hayat MJ, Howlader N, Reichman ME, Edwards BK. Cancer statistics, trends, and multiple primary cancer analyses from the Surveillance, Epidemiology, and End Results (SEER) Program. Oncologist 2007;12:20-37.

19. Mehdi I, Shah AH, Moona MS, Verma K, Abussa A,
Elramih R, et al. Synchronous and metachronous malignant tumours expect the unexpected. J Pak Med Assoc 2010;60:905-9.

20. Triantafillidou K, Dimitrakopoulos J, Iordanidis F, Koufogiannis D. Management of adenoid cystic carcinoma of minor salivary glands. J Oral Maxillofac Surg 2006;64:1114-20.

21. Norberg-Spaak L, Dardick I, Ledin T. Adenoid cystic carcinoma: Use of cell proliferation, BCL-2 expression, histologic grade, and clinical stage as predictors of clinical outcome. Head Neck 2000;22:489-97.

22. Dy GK, Miller AA, Mandrekar SJ, Aubry MC, Langdon RM Jr, Morton RF, et al. A phase II trial of imatinib (ST1571) in patients with c-kit expressing relapsed small-cell lung cancer: A CALGB and NCCTG study. Ann Oncol 2005;16:1811-6.

23. Dori S, Vered M, David R, Buchner A. HER2/neu expression in adenoid cystic carcinoma of salivary gland origin: An immunohistochemical study. J Oral Pathol Med 2002;31:463-7.

24. An J, Park S, Sung SH, Cho MS, Kim SC. Unusual expression of thyroid transcription factor 1 and napsin a in metastatic adenoid cystic carcinoma of extrapulmonary origin in the lung. Am J Clin Pathol 2014;141:712-7.

25. Giannini PJ, Shetty KV, Horan SL, Reid WD, Litchmore LL. Adenoid cystic carcinoma of the buccal vestibule: A case report and review of the literature. Oral Oncol 2006;42:1029-32.

26. Spiro RH, Huvos AG. Stage means more than grade in adenoid cystic carcinoma. Am J Surg 1992;164:623-8.

27. Rapidis AD, Givalos $\mathrm{N}$, Gakiopoulou $\mathrm{H}$, Faratzis G, Stavrianos SD, Vilos GA, et al. Adenoid cystic carcinoma of the head and neck. Clinicopathological analysis of 23 patients and review of the literature. Oral Oncol 2005;41:328-35.

28. da Cruz Perez DE, de Abreu Alves F, Nobuko Nishimoto I, de Almeida OP, Kowalski LP. Prognostic factors in head and neck adenoid cystic carcinoma. Oral Oncol 2006;42:139-46.

29. Prokopakis EP, Snyderman CH, Hanna EY, Carrau RL, Johnson JT, D'Amico F. Risk factors for local recurrence of adenoid cystic carcinoma: The role of postoperative radiation therapy. Am J Otolaryngol 1999;20:281-6.

30. Pinakapani R, Nallan C, Reddy L, Srujana Y, Mamatha B, Waghray S. Adenoid cystic carcinoma of the head and neck - Literature review. Qual Prim Care 2015;23:309-14. 